\title{
Fully autonomous planning and obstacle negotiation on rough terrain using behavior maps
}

\author{
Christian Dornhege and Alexander Kleiner \\ Institut für Informatik \\ University of Freiburg \\ 79110 Freiburg, Germany \\ \{dornhege, kleiner\}@informatik.uni-freiburg.de
}

\begin{abstract}
To autonomously navigate on rough terrain is a challenging problem for mobile robots, requiring the ability to decide whether parts of the environment can be traversed or have to be bypassed, which is commonly known as Obstacle Negotiation (ON). In this video we show the robot's ability to map, detect and negotiate obstacles. The first part shows the robot exploring a test arena, that contains a pallet and a ramp, that have to be traversed. The second part demonstrates the autonomous stair climbing skill.

The planning process uses a new concept called behavior maps, that has been developed to support planning while integrating obstacle negotiation.
\end{abstract}

\section{INTRODUCTION}

To autonomously navigate on rough terrain is a challenging problem for mobile robots, requiring the ability to decide which parts of the environment can be traversed or have to be bypassed, which is commonly known as Obstacle Negotiation (ON) [?]. While this problem has been mainly addressed in the context of semi-rough terrain, e.g. within outdoor [?], [?], [?], and indoor scenarios [?], rough terrain, containing steep structural elements, demands robots to be explicitly aware of their context, thus allowing to execute specific skills with respect to the situation.

We developed a planning framework that extends ON to the general case, where different types of terrain classes directly map to specific robot skills, such as climbing stairs and ramps. The framework is based on a new concept called behavior maps, which is utilized for the planning and execution of complex skills on rough terrain. This video demonstrates the use of behavior maps for planning of obstacle negotiation and plan execution. It shows the robot's ability to map, plan and overcome pallets and ramps during online exploration as well as the robot's ability to drive stairs.

\section{HARDWARE PLATFORM}

Figure ?? shows the tracked Lurker robot, which is based on the Tarantula R/C toy. Although based on a toy, this robot is capable of climbing difficult obstacles, such as stairs, ramps, and random stepfields. The base has been heavily modified in order to enable autonomous operation. We added sensors for measurement of the angular position of the flippers and applied touch sensors

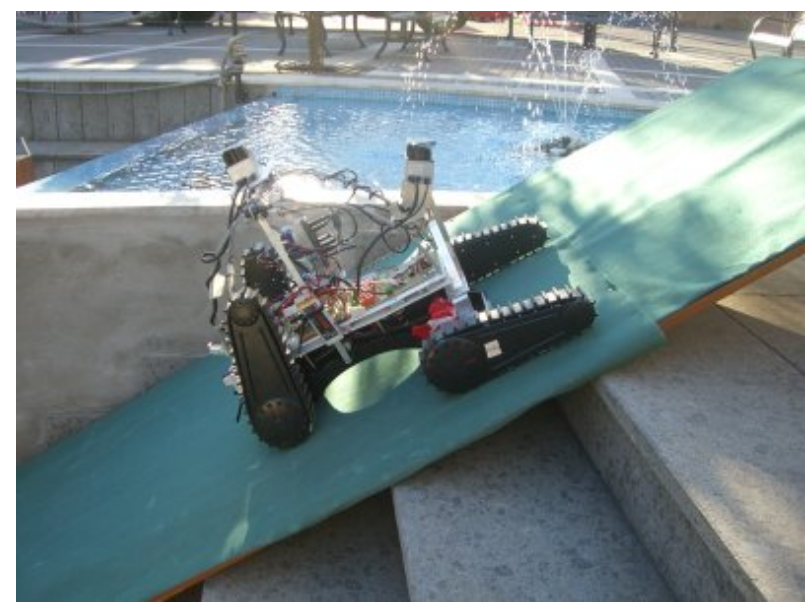

Fig. 1. The Lurker robot climbing up a ramp during the Rescue Robotics Camp 2006 in Rome.

to each flipper, allowing the robot to measure force when touching the ground or an object.

Furthermore, the robot is equipped with a 3-DOF Inertial Measurement Unit (IMU) from Xsens, allowing drift-free measurements of the three Euler angles yaw, roll, and pitch, and two Hokuyo URG-X004 Laser Range Finders (LRFs), one for scan matching, and one for elevation mapping, which can be tilted in the pitch angle within $90^{\circ}$.

\section{BEHAVIOR MAPS}

In this section we describe the use of behavior maps for online planning and skill execution. Behavior maps are directly generated from elevation maps, i.e. twodimensional grids storing in each cell the corresponding height of the terrain surface [?], [?], and a set of skill descriptions. Skill descriptions contain a set of fuzzy rules for the classification of structures they can handle, a set of spacial constraints encoding preconditions required for their execution, a cost function utilized for $\mathrm{A}^{*}$ planning on the map and the skill routine to be executed. According to these skill descriptions, elevation maps are segmented into different regions, whereas each region corresponds to a skill that can be executed therein. We utilize Markov Random Field (MRF) models, which are automatically constructed from the set of fuzzy rules, 
for detecting structure elements on the elevation map. Furthermore, behavior maps are augmented with preconditions, such as starting locations and angles, for each detected transition edge, that are automatically generated from the sets of spacial constraints. The resulting $2 \mathrm{D}$ representation encodes context information of the environment and can efficiently be utilized for the planning and execution of skills. Planning on behavior maps is done using conventional $\mathrm{A}^{*}$ planning, whereas the only distinction lies in the fact, that the plan might traverse different classes of terrain only, if the plan leads over a transition edge. Skill execution itself is handled by just calling the appropriate skill routine that is stored in the behavior map at the robot's position. The final system consists of four main modules, which are all executed in real-time during navigation: elevation mapping, terrain classification, skill planning, and motion control.

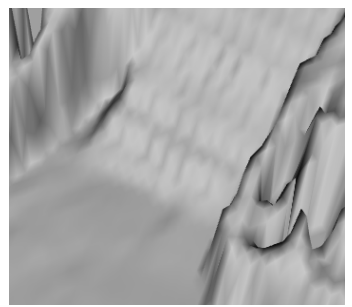

(a)

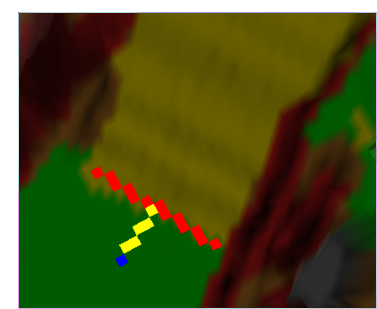

(b)
Fig. 2. Visualization of the determined skill preconditions: (a) Elevation map with ramp structure. (b) Classified elevation map with the detected classes floor (green), ramp (yellow), and walls (dark red), detected transition edge (red), and detected preconditions, which are the starting point (blue) and staring direction (yellow).

\section{VIDEO CONTENT}

The presented video consists of two parts. The first part shows a fully autonomous run in a test arena to demonstrate the system's ability of online mapping while performing planning and obstacle negotiation during an exploration task over rough terrain. We focus the video on the decisions that the robot does while performing exploration. These are aimed to keep the robot exploring floor areas as long as possible while collecting information about detected obstacles. The robot should decide to surpass an obstacle only after a floor area is fully explored.

Figure ??(b) shows the exploration trajectory. The robot deliberately decided to explore each segment of the map before climbing an obstacle to reach another area. During this exploration run the robot traversed a trajectory with a length of $23.00 \mathrm{~m}$ within 9 minutes and 55 seconds. We included the full trajectory in the video by speeding up some parts, being able to show decision points and the corresponding behavior maps in more detail.

Reliable execution of complex autonomous skills like stair climbing are a necessity for exploration on rough terrain. Therefore in the second part of the video we (a)

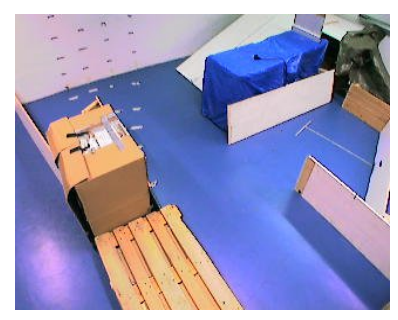

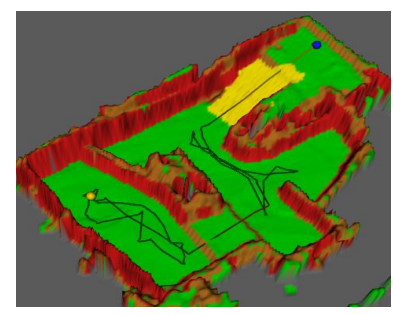

(b)
Fig. 3. (a) Test arena for the exploration. (b) Classified map containing the trajectory of the exploration. The robot started exploring the area at the left, crossed the pallet, continuing to explore the center area and finally drove up the ramp.

show the robot climbing a stairwell utilizing its arm angular sensors, touch point sensors in the arms and pitch and roll angles from the IMU. The robot is able to conquer the whole stairwell without human interaction. This is not accomplished by using prerecorded motion sequences, but by using the robot's sensors to become aware of the current state, leading to a stable execution of the skill over the whole distance of the stairwell without becoming misaligned or stuck. In the case of the stair climbing skill, after lifting up the first stair with the front flippers, the robot mainly uses its touch sensors in the front flippers to determine, where they have contact with the step and based on that adjusts the flipper angles. The rear flippers are used to keep a constant pitch during the skill execution. Finally the end of the stairwell is detected automatically and the robot switches back into driving position to enable normal ground exploration.

\section{ACKNOWLEDGMENTS}

The authors gratefully acknowledge the work done by all members of the RescueRobots Freiburg team, particularly Rainer Kümmerle and Bastian Steder. 